\title{
Levitation of liquid microdroplets over a dry heated substrate near triple-phase contact line
}

\author{
Dmitry Kirichenko ${ }^{1,2,}$, Dmitry Zaitsev ${ }^{1,2}$, and Oleg Kabov ${ }^{1,2}$ \\ ${ }^{1}$ Kutateladze Institute Institute of Thermophysics, 630090 Novosibirsk, Russia \\ ${ }^{2}$ Novosibirsk State University, 630090 Novosibirsk, Russia
}

\begin{abstract}
Levitating droplets of liquid condensate are known to organize themselves into ordered arrays over hot liquid-gas interfaces. We report experimental observation of similar behaviour over a dry heated substrate in the vicinity of the triple-phase contact line with high local evaporation rate. It was found that there is an area near the contact line, where no levitating droplets are observed. The width of this area increases with the substrate temperature. Also, it was found that the distance between adjacent levitating droplets increases with the temperature.
\end{abstract}

\section{Introduction}

One of effective solutions for removing high heat fluxes are systems based on two-phase flows and sprays [1-3]. The effectiveness of these methods can be impaired by the Leidenfrost effect [4]. Liquid may not reach the heating surface, which can affect the cooling capacity. The behavior of liquid microdroplets falling on a solid surface heated below the Leidenfrost temperature (about $200{ }^{\circ} \mathrm{C}$ for water) is not well understood. Droplet levitation over hot liquid-gas interfaces [5-6] can have a significant effect on the performance of twophase cooling systems. Experimental studies of microdroplets levitation over a dry solid surface with relatively low temperature have been conducted in the works [7-10].

A number of applications such as spray cooling [1], heat pipes [11], film rupture [12] involve contact lines; that is, lines where the liquid-vapor or liquid-air interface comes into contact with the solid wall. We note that macroscale observations do not always allow one to determine whether the solid wall is actually dry or covered by an ultrathin adsorbed film. The term "contact line" is often used for the latter situation even though the true contact of the fluid interface with the solid wall is not achieved; some authors prefer the term "apparent contact line" to describe this situation. In [13-14], the behavior of levitating droplets was studied when they transit from hot liquid interface to dry area. It was found that when approaching the contact line, the droplet levitation height increases several times, which indicates the existence of intense evaporation in the region of the contact line.

\footnotetext{
*Corresponding author: Dmitriy_Kirichenko@mail.ru
} 


\section{Experimental technique}

A sketch of the experimental setup is shown in Fig. 1. The test section is a stainless steel plate of $40 \times 40 \times 5 \mathrm{~mm}^{3}$ in size with a flush-embedded $10 \times 10 \mathrm{~mm}^{2}$ copper rod, electrically heated by a nichrome wire. According to the measurements, the condition of constant temperature, denoted by $\mathrm{T}_{\mathrm{w}}$, is satisfied along the copper surface. The test section is installed horizontally and open to the atmosphere; the temperature of ambient air is $25 \pm 2^{\circ} \mathrm{C}$. Optical recording was made with a high-speed camera FAST- CAM SA1.1 (5.400 fps, resolution of $1.024 \times 1.024$ pixels) coupled with a microscope objective through a set of extension tubes and bellows.

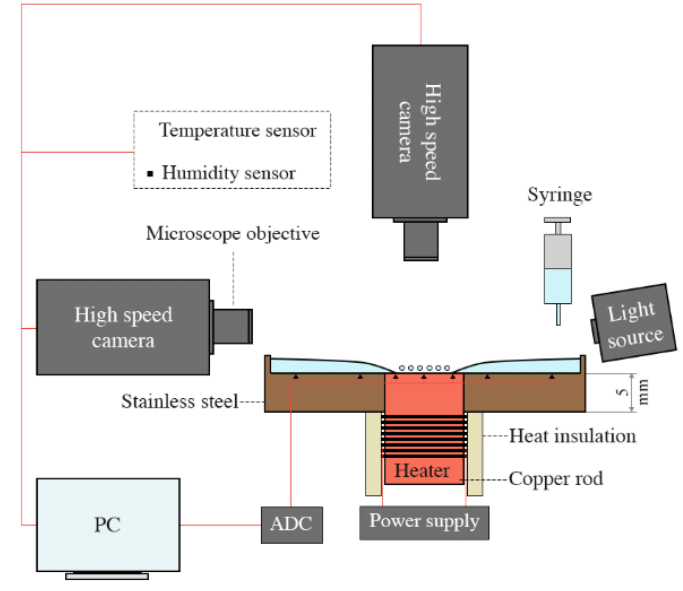

(a)

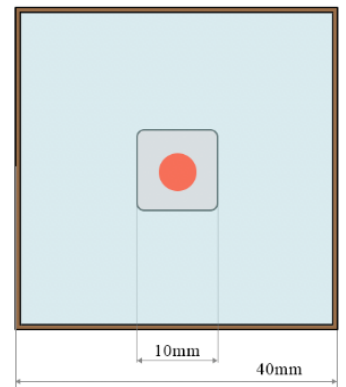

(b)

Fig. 1. (a) Sketch of the side view of the experimental set-up; (b) sketch of the top view of the test section.

Working liquid in the experiments is ultra-pure degassed distilled water (Merck Millipore) with the initial temperature of $25^{\circ} \mathrm{C}$. Wetting properties of the surface are important for obtaining the steady configuration needed for our experimental study (a dry patch in a liquid layer, as described in more detail below) so particular attention was paid to control and characterization of surface properties. To obtain the desired wetting characteristics, the working surface of the test section was rough polished prior to the start of the experiment. The morphology of the surface was analyzed using scanning electron microscope (JEOL JSM6700F) and atomic force microscope (Solver Pro NT MDT). The root mean square (RMS) surface roughness was found to be $0.50 \mu \mathrm{m}$. The equilibrium contact angle for water on the copper surface was measured at different points by the method of a sessile liquid drop at the room temperature of $25 \pm 2^{\circ} \mathrm{C}$. To obtain the profile of the drop surface, the shadow method was used with a collimated light source and digital camera Nikon D800 having resolution of 6 micron/pixel. The resulting images were processed using the software by KRUSS. The advancing static contact angle measured at different points over the surface of the copper heater was $74 \pm 9^{\circ}$, whereas the receding contact angle was close to zero (less than $10^{\circ}$ ).

\section{Results and discussions}

At the start of the experiment, the substrate is covered by a uniform layer of ultrapure water of thickness of $0.4 \mathrm{~mm}$. A dry patch, shown in the sketch in Fig. 2a, is formed in the liquid layer by a downward pulsed jet of air. The heater surface is sufficiently rough to ensure pinning of the contact line, so the dry patch created by the air jet persists throughout the 
duration of the experiment. Levitating droplets are shown near the right boundary of the Fig. 2a. These droplets are part of a larger ordered array. As they approach the contact line, the local gas flow velocity increases due to stronger evaporation and the resulting force on the droplet becomes higher than its weight, resulting in an upward deflection of the droplet trajectory. However, as the droplet finds itself over the dry patch, it starts moving downward until it reaches a location very close to the solid surface. These processes were recorded by the high-speed camera; typical video frames are shown in Fig. $2 b$ (the side view and the top view correspond to different experimental runs because the camera position had to be changed to obtain the top view Fig. 2a). As the droplets find themselves in the region above the hot dry surface, they disappear by evaporation within about a second. We have conducted experimental investigations of interaction of arrays of levitating droplets with a region of high evaporation near the contact line on a heated surface. Droplets are seen over the solid surface except in the vicinity of the contact line, where there is a narrow region near the contact line where no levitating droplets are observed. This forbidden zone is clearly seen in both the side-view and top-view photographs in Figure 2a. Its width is between 10 and 50 $\mu \mathrm{m}$ and typically increases with temperature, which can be explained by the higher evaporation rate. Fig.3 shows the dependence of width of "forbidden zone" on temperature of the substrate. Dependence of interdroplet distance on the substrate temperature have also been obtained, Fig. 4. Interdroplet distance (distance between centers of two adjacent drops) have been measured using top view experimental photos and also was found to increase with temperature.

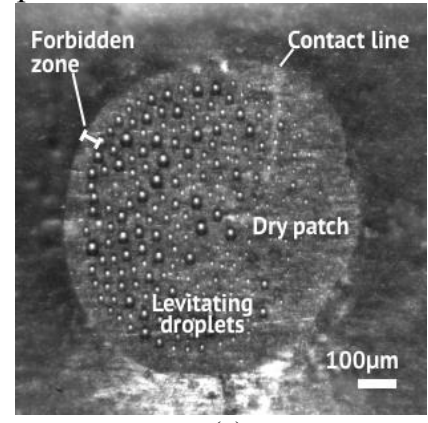

(a)

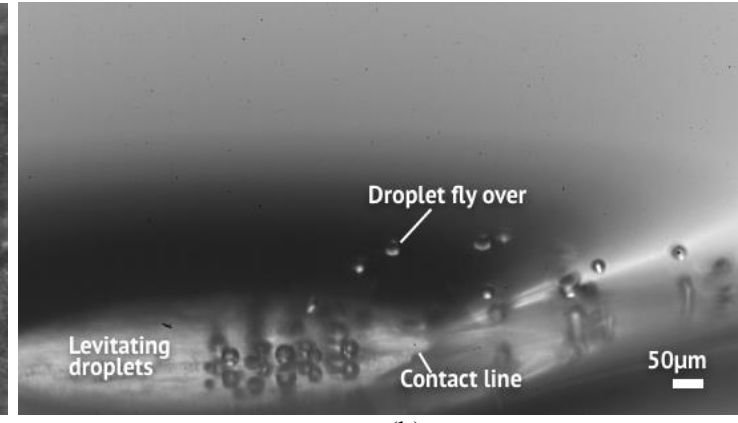

(b)

Fig. 2. Experimental photo: (a) top view of a dry patch and an array of ordered levitating droplets for the substrate temperature $90^{\circ} \mathrm{C}$; (b) side view of the dry patch.

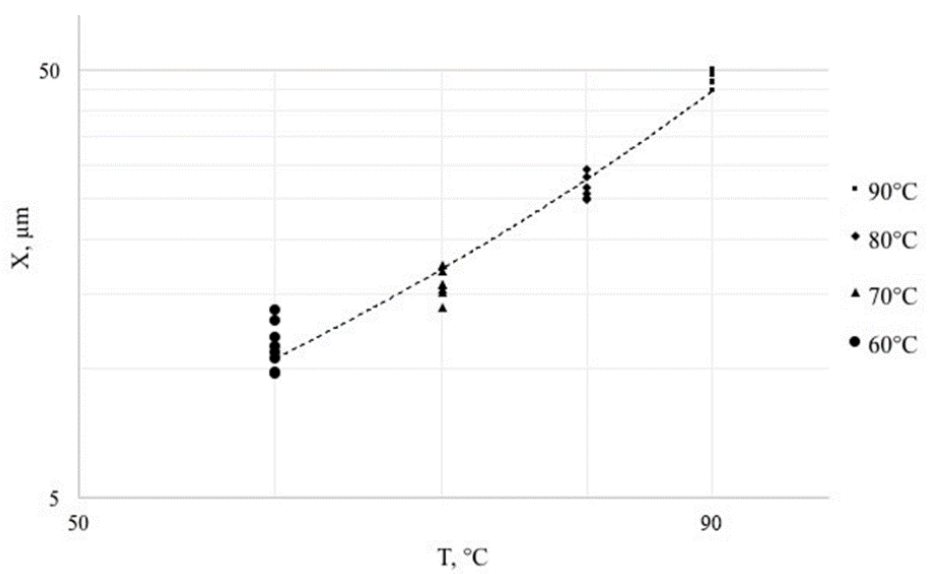

Fig. 3. Dependence of width of "forbidden zone" on substrate temperature. Dashed line generalization of all data. 


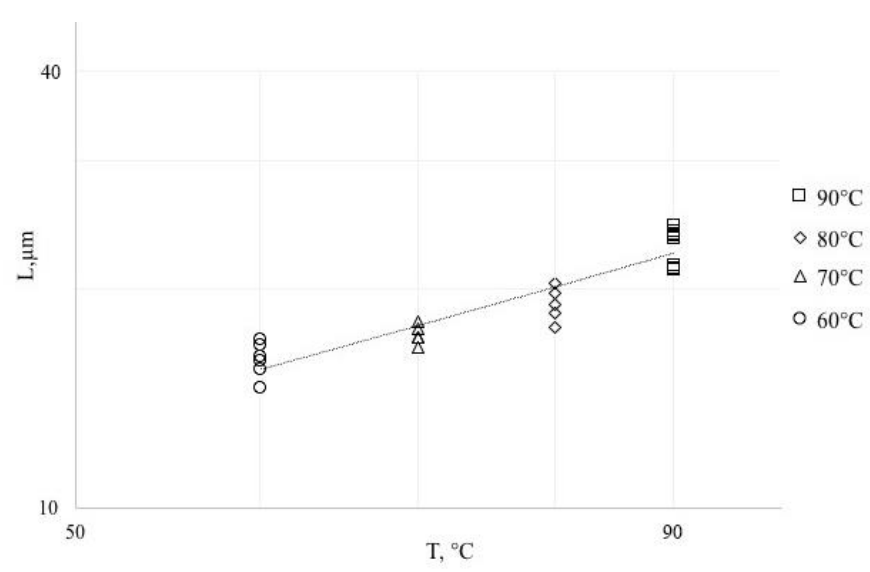

Fig. 4. Dependence of interdroplet distance on substrate temperature. Dashed line - generalization of all data.

We have conducted experimental investigations of interaction of arrays of levitating droplets with a region of high evaporation near the contact line on a heated surface. It was found that there is an area near the contact line, where no levitating droplets are observed. The width of this area increases with the substrate temperature. Also, it was found that the distance between adjacent levitating droplets increases with the temperature.

The study was financially supported by the grant of Russian Foundation for Basic Research (agreement No. 17-38-50222).

\section{References}

1. J. Kim, Int. J. Heat Fluid Flow 28, 753 (2006)

2. R. Srikar, T. Gambaryan-Roisman, C. Steffes, P. Stephan, C. Tropea, A. L. Yarin, Int. J. Heat Mass Transfer 52, 5814 (2009)

3. O. A. Kabov, D. V. Zaitsev, V. V. Cheverda, A. Bar-Cohen, Exp. Therm. Fluid Sci. 35, 825 (2011)

4. M. Shirota, M. A. J. van Limbeek, C. Sun, A. Prosperetti, D. Lohse, Phys. Rev. Lett. 116, 064501 (2016)

5. A. A. Fedorets, JETP Lett. 79, 372 (2004)

6. A. A. Fedorets, I. Marchuk, and O. Kabov, Tech. Phys. Lett. 37, 116 (2011)

7. D. V. Zaitsev, D. P.Kirichenko, V. S. Ajaev, O. A. Kabov, Phys. Rev. Lett. 119, 94503 (2017)

8. D. P. Kirichenko, D. V. Zaitsev, O. A. Kabov, MATEC Web Conf. 72, 01046 (2016)

9. D. V. Zaitsev, D. P. Kirichenko, MATEC Web Conf. 84, 00044 (2016)

10. D. P. Kirichenko, D. V. Zaitsev, O. A. Kabov, J. Phys. Conf. Ser. 754, 042008 (2016)

11. A. Chatterjee, J. L. Plawsky, P. C. Wayner, Adv. Colloid Interface Sci. 168, 40 (2011)

12. D. V. Zaitsev, A. A. Semenov, O. A. Kabov, T and A 23, 625 (2016)

13. O. A. Kabov, D. V. Zaitsev, D. P. Kirichenko, V. S. Ajaev, Interfacial Phenomena and Heat Transfer 4, 207 (2016)

14. O. A. Kabov, D. V. Zaitsev, D. P. Kirichenko, V. S. Ajaev, Nanoscale and microscale thermophysical engineering 21, 60 (2017) 